\title{
Matematización de los procesos químicos. Segunda parte
}

Recibido: 13-08-2010 | Aceptado:20-12-2010

Mathematization of Chemical processes. Part II.

Rómulo Gallego Badillo*

Royman Pérez Miranda**

Adriana Patricia Gallego Torres
Resumen: En esta segunda parte -ya anunciada en la primera- se analizará histórica y epistemológicamente la creación de la fisicoquímica como disciplina, ya que es en sus formulaciones donde se introduce una matemática superior al álgebra, que fue el fundamento de la construcción de la química estructural o química orgánica, en la segunda mitad del siglo XIX. Se resaltarán, igualmente, los personajes que participaron y desarrollaron esta disciplina en el periodo mencionado. A manera de conclusión, se presentarán también las reflexiones didácticas indispensables.

Palabras clave: historia, epistemología, fisicoquímica, didáctica.

\begin{abstract}
In this second part of the study the creation of physical chemistry is analyzed historically and epistemologically like a discipline, as it is in its formulations in which a higher math superior to algebra is introduced as the basis for the construction of structural or organic chemistry, in the second half of the nineteenth century. It also highlights the scientists involved and recognized in the same period of time, regarding this discipline. To conclude, we will also present essential teaching reflections.
\end{abstract}

Keywords: History, Philosophy, Physical Chemistry, Didactics.

* Profesor de la Universidad Pedagógica Nacional. Bogotá, D. C. Colombia. rgallego@pedagogica.edu.co

** Profesor de la Universidad Pedagógica Nacional. Bogotá, D. C. Colombia. royman@pedagogica.edu.co

*** Profesora de la Universidad Distrital Francisco José de Caldas. Bogotá, D. C. Colombia. adpgallegot@udistrital.edu.co 


\section{Introducción}

En la primera parte se finalizó con las leyes de la termodinámica, puesto que se afirmó que fue la conversión de los procesos químicos en sistemas termodinámicos la que posibilitó, por primera vez, la introducción de las ecuaciones diferenciales en las explicaciones químicas, específicamente para el análisis de los intercambios de energía en esos procesos. Se aludió a J. E. van't Hoff (18521911), matemático de formación interesado en estas temáticas, a W. Ostwald (1853-1932) y a S. Arrhenius (1859-1927), químicos ambos, quienes son los responsables de la creación de la fisicoquímica como disciplina (Gallego BadiIlo, Pérez Miranda y Figueroa Molina, 2011).

De la misma manera, se anotó que la introducción de las ecuaciones diferenciales chocó contra la tradición de los afamados químicos, cuya formación matemática no pasaba del algebra y, por tanto, no podían acceder al contenido significativo de este lenguaje, alegando además, que carecía de soportes experimentales (Brock, 1998). Como se sabe, es A. L. Lavoisier (1743-1794) quien acude al algebra con la formulación de la ecuación química como una analogía de la balanza de dos platillos y la formulación de la Ley de la conservación de los pesos; de ahí que la ecuación química sea algebraica y fuera el primer método para balancearla, según lo mandado por la ley citada (Schneer, 1975; Bensaude-Vincent y Stengers, 1997; Fara, 2009).

Anótese que es la creación de la fisicoquímica la que obliga, en las primeras décadas del siglo $X X$, a una reforma del plan de estudio para la formación profesional de los químicos establecido por J. von Liebig (1803-1873) con miras a una formación matemática superior, para lo cual se redactaron textos que llevaron el nombre genérico de matemáticas para químicos.

\section{Un repaso a la prehistoria}

Se ha establecido que la prehistoria comprende la Edad de piedra (dividida por los historiadores en periodo paleolítico -determinado por el uso de la piedra tallada- y neolítico -uso de piedra pulimentada-) y la Edad de los metales.) Sin mayores consideraciones, se dice que la edad de los metales está dividida en tres periodos: el periodo del cobre (4000-2500 a. C.), el del bronce (3000-800 a. C.) y el del hierro (siglo XIV al III a. C.) Esta secuencia lineal suele dejar de lado lo que significa para el proceso de humanización y de concepción, el hecho de que fuera factible actuar sobre la materialidad del mundo.

En la síntesis anotada, es común que no se haga referencia a esa actividad creativa, mediante la cual un grupo de seres humanos inventaron la alfarería. Ello significó un proceso paulatino de perfeccionamiento de técnicas, quizás por ensayo y error, en el que fue indispensable identificar aquella arcilla que había que recolectar, para que sometida a los procesos de cocción, se dispusiera de utensilios que, además de elaborados, superaran los de la piedra pulida.

Más allá de las referencias lineales, circunscribiéndonos a la actividad delimitada por la alfarería, es posible que ella diera origen al convencimiento de que estos artesanos -seres humanos de la prehistoria- se hallaban en condiciones de transformar, de incidir en la materialidad de aquello que los rodeaba. No obstante, la transformación de la arcilla mediante el fuego con el fin de obtener utensilios y otras obras, es claro que no los separaba significativamente del material inicial: esa arcilla cocida seguía siendo arcilla, aun cuando de diferente color del inicial. Se piensa que esta experiencia continuada hizo posible el desarrollo del horno, y más allá, el de los crisoles, como el recurso experiencial que desembocará en la metalurgia. 
La edad de los metales surgió con la obtención de cobre metálico. Las hipótesis de que fue un hallazgo fortuito, que hablan a favor de que un trozo de azurita arrojado a un fogón de manera casual produjo el chorro de cobre metálico, no se han podido demostrar experimentalmente (Javanovic, 1980). En efecto, el procedimiento más simple para la obtención de cobre metálico consiste en calentar intensamente malaquita finamente pulverizada en una copa de arcilla cubierta con una vasija (Levi-Straus, 1964). De todas maneras, sean malaquita o azurita los minerales para la obtención de cobre metálico, es el conocimiento del hombre alfarero el que se halla en la base para ese salto artesanal requerido para hacerse metalurgista.

En esta nueva etapa, ha de ir más allá del conocimiento de los materiales iniciales y su recolección, para pasar a un tratamiento nuevo en el que el material de trabajo ha de ser obtenido por una operación que se diferencia de la propia ejecutada en la alfarería. De alguna manera, las vasijas de barro cargan con la semejanza de su origen. Por el contrario, el cobre obtenido como material de trabajo, dista del mineral del que se obtiene. El ser humano, como metalurgista, desarrolla el poder de intervenir en la naturaleza de otra manera innovadora; una intervención que genera poder, y por tanto, el procedimiento artesanal ha de ser elevado a la categoría de secreto propio de un gremio.

De hecho, ese procedimiento será explicado por la mentalidad propia de ese entonces; explicaciones que serán de carácter mágico, en el sentido original de esta palabra. Es magia, por cuanto el artesano metalurgista ha adquirido el poder de transformar la materialidad de su entorno. Es el lenguaje en el que expresan dichas explicaciones, el que será la base del arte que se denominará después como alquimia (Federmann, 1974; Schneer, 1975). Desde su origen cargará con esa doble naturaleza: ser productiva, a la vez que ser un secreto de un gremio que, con el tiempo, se convertirá en corporación (Manocorda, 1987).

Vendrá después a reforzar ese convencimiento, primero, la operación metalúrgica de la aleación con el estaño, para dar origen a la edad del bronce. Desde esta, la obtención del hierro metálico. Si es factible transformar los minerales malaquita y azurita en cobre metálico -algo que no es evidente en la apariencia física de dichos materiales-, entonces ese nuevo arte ha de posibilitar convertir cualquier metal no noble en oro.

Sin embargo, el origen de la alquimia propiamente dicha, se ubica en la época del esplendor de Alejandría y su cultura helenística; espacio este en el que confluyeron la filosofía griega y las doctrinas místicas orientales. Se hará objeto de estudio la alquimia árabe, que pasará a Europa Occidental, donde sufrirá la influencia de las ideas aristotélicas y cristianas, para luego ser dejada definitivamente de lado con la iniciación de una química basada en la aproximación epistemológica positivista de A. Comte (Brock, 1998; Bensaude-Vincent y Stengers, 1997; Fara, 2009; Ihde, 1984; Sánchez, 2009). Se disertará más adelante acerca del empleo de la categoría de ciencia o de arte, para enclavarla histórica y epistemológicamente.

La combustión siguió siendo objeto de explicaciones de toda índole. Una de ellas fue elaborada por G. E. Stahl (1660-1734), quien fue primero alquimista y luego se dedicó a la práctica de la medicina. Partiendo de las ideas de J. J. Becher (1635-1682) respecto a las tres tierras, se apoyó en la de la terra pinguis o combustible, a la que denominó flogisto, de la palabra griega "phlogistos" 
que significa inflamable (Schneer, 1975). Su modelo explicativo para la combustión y la calcinación, dada su elegancia y lógica interna, fue la primera y única "teoría verdaderamente química" (Izquierdo, 1988), hasta el punto de que los más famosos practicantes anteriores a Lavoisier la adoptaron; entre ellos cabe mencionar a C. W. Scheele (17421786), J. Priestley (1733-1804), H. Cavendish (1731-1810) y J. Black (1728-1799) (Laín y López, 1963).

Toda la comunidad se hallaba satisfecha con el modelo del flogisto, a pesar de los problemas que presentaba en relación con la contabilidad de los pesos, problemas que eran explicados acudiendo a hipótesis ad hoc (Lakatos, 1983). Vendrá Lavoisier quien, proponiéndose ser el Newton de la química, demostrará que sus fundamentos carecían de soporte experimental, durante una conferencia que pronunció en la Academia, el 15 de abril de 1775 y que tituló "Acerca de la naturaleza del principio que se une con los metales en la calcinación y les hace aumentar de peso" (Lockemann, 1960). Surgió el modelo del oxígeno o de la oxidación. T. S. Kuhn (1972) y B. Bensaude-Vincent (1991), suelen conceptualizar este hecho como ejemplo de "revolución científica". Sin embargo, de los aportes de Lavoisier, no quedó para la historia sino la Ley de la conservación de los pesos (Bowler y Morus, 2007); ni siquiera su propuesta de lo que debería ser un experimento en química.

\section{De la fisicoquímica}

La creación de esta disciplina constituyó un capítulo aparte en la historia de la construcción de la química, cuyos resultados estuvieron y han estado ligados a la ingeniería, principalmente, aun cuando sus contrastaciones revertieron en la comprensión de los procesos ya delimitados por quienes, de manera exitosa, construyeron la química orgánica o química estructural, sin la necesidad conceptual y metodológica de ocuparse de los problemas de las relaciones energéticas implicadas en ellas.

Aún cuando se ha resaltado la introducción de la termodinámica clásica para entender los orígenes de esta disciplina, la formulación inicial de la fisicoquímica tendría que ser inscrita en otra perspectiva, por lo que habría que aludir a C. F. Wenzel (1740-1793) y a C. L. Berthollet (1748-1822), quienes fueron los que se preguntaron por la relación entre la masa química y la acción química, sin llegar a un modelo admisible; en otras palabras, por qué y cómo ocurren las reacciones químicas. Luego, en 1867, contribuirán a este esclarecimiento C. M. Guldberg (1836-1902) y P. Waage (1833-1900), que delimitarán el problema en términos de la Ley de acción de masas, a la vez que inscribirán los procesos químicos en el espacio y el tiempo, para hablar de la velocidad de las reacciones y sugerir el equilibrio químico (Lockemann, 1960). No obstante, es J. H. van't Hoff, matemático de formación, iniciador de la dinámica química -disciplina que más tarde pasó a denominarse cinética química- quien expuso la primera matematización en 1884, en su libro "Estudios de dinámica química", significativo para la posterior formulación y desarrollo de lo que será la termodinámica química (Laidler, 1995).

Es un hecho histórico y menester resaltar, que la creación de la fisicoquímica no obedecerá, en sentido estricto, a la lectura desde la termodinámica clásica de los procesos químicos, la que de manera aislada posibilitará la construcción de la fisicoquímica como disciplina académica, dado que este giro conceptual y metodológico será producto de la pregunta ¿cómo ocurren?, cómo y de qué manera se llevan a cabo. Ello significó una introducción en la naturaleza 
específica del quehacer de los químicos, de esa razón de ser que desembocaría en el dominio definitivo de aquella convicción y actitud que venía desde los alquimistas artesanos, de que el ser humano podía intervenir creativamente sobre la naturaleza para transformarla; un proyecto que ya había dado sus frutos con la creación y desarrollo de la química estructural (Gallego Badillo, Pérez Miranda, Gallego Torres, y Torres de Gallego, 2006).

Retomando el rumbo inicial, es preciso delimitar que en los inicios de la fisicoquímica como disciplina, más allá de la termodinámica clásica en la que se fundamentó y de conformidad con las especificidades de sus creadores, fueron ellos quienes se ocuparon del problema de la cinética química, y no de la termodinámica clásica en sí, que ya estaba para entonces creada. Por tanto, el origen de esta disciplina académica hay que encontrarlo en la constitución de dicha problemática de conocimiento.

Esto ocurrió en los inicios de 1865 debido a la colaboración que establecieron el químico G. V. Harcourt (1834-1919) y el matemático W. Esson (1838-1916), este último, quien hizo de la cinética su campo de saber de mayor interés. Esson, acudió a distintos planteamientos con las ecuaciones diferenciales para formular diferentes tipos de reacciones. Las integró entre los límites del aumento de la cantidad de productos en función del tiempo, de tal manera que se convenció de que se encontraba en una problemática admisible. Como resultado de sus trabajos, fue él quien acuñó las expresiones que en la actualidad se conocen como reacciones de primer, de segundo y de tercer orden, de conformidad con la concentración de los reactantes, denominación que introdujo W. Ostwald en 1887 (Laidler, 1995).

Es entonces W. Esson a quien hay que atribuirle ser el verdadero introductor de las matemáticas en este nivel del discurso químico en general, y en el de la nueva disciplina que se estaba creando. Establece además el concepto de mecanismos de reacción, que se desprenden del análisis matemático de la cinética química. Ha de haber claridad en que los análisis termodinámicos de los procesos de intercambio de energía y de producción de trabajo mecánico pertenecen a los físicos. Pero aquellos acerca de cómo se suceden estos procesos, desde las perspectivas de los intercambios energéticos, es propio de los ingenieros químicos.

Anótese que en el libro ya citado de van't Hoff, este investigador amplía y generaliza los análisis matemáticos de Harcourt y Esson. Van't Hoff empleó para el orden de las reacciones, los términos de monomolecular y bimolecular, que representan la dependencia relativa de la velocidad de reacción con respecto a la concentración. Obsérvese que en la actualidad los químicos emplean los conceptos de unimolecular, bimolecular y trimolecular para señalar el número de moléculas que intervienen en una reacción particular (Laidler, 1995). Como ejemplo de una reacción monomolecular para formular la correspondiente ecuación diferencial de la velocidad, acudió a la descomposición de la arsina en fase gaseosa, planteándola como sigue:

$-(\mathrm{dC} / \mathrm{dt})=\mathrm{kc}$

Donde "k" es la constante de velocidad.

Van't Hoff, en su libro Estudios, introdujo el denominado método diferencial para analizar los resultados cinéticos y determinar el orden de una reacción, de acuerdo con la medición de la velocidad " $v$ " a diferentes concentraciones " $c$ " del reactante:

$\mathrm{v}=\mathrm{kc} \mathrm{c}^{\prime \prime}$ 
Un problema que duró sesenta años para ser resuelto, fue el de la dependencia de la velocidad con respecto a la temperatura " $\mathrm{T}$ ", que tempranamente, en 1850, fue expresado por L. F. Wilhelmy (1812-1864), aunque que no fue satisfactorio. En la solución de este problema también es reconocido el aporte dado en su libro de 1884, en el que se ocupa del equilibrio químico y propone la constante " $K$ " respectiva, recogidos en la ecuación:

$(\mathrm{d} \ln \mathrm{K}) / \mathrm{dT}=\mathrm{Q} / \mathrm{RT}^{2}$

" $R$ " es la constante de los gases y " $Q$ " el calor absorbido por el sistema químico si la reacción ocurre a presión constante; en consecuencia, y dado que $\Delta \mathrm{H}^{0}=\mathrm{Q}_{\mathrm{p}}$ el cambio entálpico producido es "flujo" de calor, entonces,

$(\mathrm{d} \ln \mathrm{K}) / \mathrm{dT}=\left(\Delta \mathrm{H}^{0}\right) / \mathrm{RT}^{2}$

La ecuación que permitirá delimitar el concepto de equilibrio químico será propuesta por J. W. Gibbs (1839-1903). Este científico fue quien, entre 1873 y 1878, construyó la termodinámica del equilibrio con su modelo de las fases. Publicó sus contribuciones así: en 1873, A method of geometrical representation of the thermodynamics properties of substances by means of surfaces; en 1876 y 1878, On the equilibrium of heterogeneous substances. Definió, igualmente la función de trabajo que lleva su nombre, conocida también como la energía libre de Gibbs, entendida en términos de diferencia de potenciales. Fijando las condiciones iniciales y finales de los parámetros termodinámicos que rigen las condiciones del equilibrio químico, se tiene que,

$\Delta \mathrm{G}^{0}=\Delta \mathrm{H}^{0}-\mathrm{T} \Delta \mathrm{S}^{0}$

Todos aquellos que se han ocupado de esta disciplina, recordarán que, si $\Delta \mathrm{G}^{0}=0$, la reacción se halla en equilibrio. Esto es, $\Delta \mathrm{H}^{0}$ $=\mathrm{T} \Delta \mathrm{S}^{0}$. Por otro lado, si $\Delta \mathrm{H}^{0}>\mathrm{T} \Delta \mathrm{S}^{0}, \Delta \mathrm{G}^{0}$ es positiva (+) y la reacción transcurre en el sentido contrario a como está planteada. Si la energía libre de Gibbs es negativa, transcurre en la dirección en la que está formulada. Se puede interferir el equilibrio, teniendo en cuenta la regla propuesta en 1884 por H. Le Chatelier (1850-1936), quien publicó sus trabajos Sur un énoncé général des lois des équilibres chimiques (1884) y Sur les lois d'équilibre chimique (1888).

La introducción de la termodinámica clásica fue, definitivamente, para un nuevo discurso químico que, desde otra matemática y con los resultados obtenidos, logró el reconocimiento social como actividad científica (Echeverría, 1998) y la correspondiente institucionalización de dicho saber (Bowler y Morus, 2007), entrando a los currículos universitarios de química y haciéndose objeto de enseñanza. Se empezaron a escribir los textos didácticos necesarios.

Para reiterarlo, el experimentalismo -de corte positivista, instaurado por J. von Liebig y posiblemente con la herencia lavoisieriana del nivel matemático propio del álgebra-es medido en la cuantificación de prácticas de los aspirantes a ser profesionales como químicos. W. Ostwald, a pesar de los logros obtenidos, desdirá de las dimensiones del trabajo científico alcanzado por los químicos orgánicos (Brock, 1998). Los creadores de la fisicoquímica, con el fin de fundar esta nueva disciplina, crearán la revista Zeitschrift für phisikalische Chemie, y en la socialización de la misma, W. Ostwald publicará en 1885 su texto "Lehrbuch der allgemeinen Chemie".

De acuerdo con lo resumido en los párrafos anteriores, resulta difícil sostener que la fisicoquímica se construyó debido a una sustitución de teorías, a un cambio paradigmático o al abandono de un programa de 
investigación que se hizo regresivo. En primer lugar, porque obedeció, como ya se ha sostenido, a la introducción de la mirada termodinámica a los procesos químicos, con la formulación de que estos debían inscribirse en el tiempo y en el espacio, lo que dio origen a la hoy cinética química.

En segundo lugar, porque la química estaba dominada por la exitosa actividad científica lograda por los químicos orgánicos, aún cuando las generaciones posteriores de científicos se apropiaran del orden y de los mecanismos de reacción derivados de las integraciones de las ecuaciones diferenciales en las que se delimitaron sus respectivas cinéticas. En consecuencia, las propuestas epistemológicas de K. Popper, T. S. Kuhn e I. Lakatos, no son suficientes para comprender epistemológicamente el desarrollo histórico de la fisicoquímica. ¿Hasta dónde se podría acudir a la propuesta de S. Toulmin (1977)? ¿Hasta dónde se podría sostener que la formulación de la fisicoquímica produjo una "revolución científica"? ¿Hubo sustitución de teorías? ¿Hubo una ruptura epistemológica? Los autores de la presente contribución son del parecer de que las respuestas a las anteriores preguntas son negativas.

\section{Los efectos histórico- epistemológicos de la creación de la fisicoquímica}

La proximidad con la física condujo a pensar que la química era ciencia, hasta el punto de que respetados analistas así lo consideraron y sostuvieron (Bachelard, 1979), postura que recientemente es afirmada (Jensen, 1998a; 1998b; 1998c), cuyas propuestas, a juicio de los autores de esta contribución, no tienen en cuenta la construcción de la química estructural; esa que se construyó con el modelo de Dalton y las leyes ponderales, mucho antes de la mecánica cuántica de matrices y ondulatoria. Es más, los químicos se han dedicado a construir moléculas nuevas, razón por la cual el objeto de saber es la "molecularidad" y en menor grado la "atomicidad" (Hoffmann, 1997).

Se reafirma que, tanto la mirada bachelardiana como la de Jensen, se fundamentan casi que en su totalidad, en las proposiciones de la fisicoquímica y no serían aplicables a la construcción de la química estructural o química orgánica. Hay que recordar aquí que las leyes ponderales, incluyendo la de la periodicidad, sostiene E. R. Scerri (1997), no obedecieron a una deducción axiomática. Sin embargo, se ha podido demostrar que no pudieron ser inducidas de un contexto netamente empírico, puesto que detrás de ellas existen representaciones, modelos sin los cuales no hubiesen sido formuladas (Gallego Badillo, Pérez Miranda y Gallego Torres, 2009). En este sentido, el "fisicalismo" del que habla E. Mayr (2006), en el caso de la fisicoquímica como disciplina, sería admisible.

\section{Hacia una didáctica de la fisicoquímica}

Hay que empezar por afirmar que reconocidos personajes han planteado, en cuanto a la química en general, que la posición epistemológica dentro de la cual se enfoca su enseñanza está equivocada (Scerri, $2001 ; 2003)$, sobre todo porque no cabría el enfoque deductivista-constructivista $y$, en este sentido, se agrega, sería insostenible. Hay que anotar que dentro de la perspectiva "fisicalista" de W. B Jensen, que ha sido citado y referenciado, existe una propuesta didáctica (Nelson, 2002), que deberían examinar quienes ejercen profesionalmente como profesores de esta disciplina académica. 
Hay que agregar que la química que se hace objeto de enseñanza en todos los niveles de los sistemas educativos, plasmada en los textos didácticos correspondientes, es una disciplina triplemente descontextualizada. En primer lugar, porque se le ha extraído del contexto comunitario en que fue construida y se da a entender que fue obra de individuos geniales; segundo, en razón de que se enseña por fuera de los problemas conceptuales y metodológicos cuyas soluciones fueron admitidas por la comunidad de especialistas; $y$, en tercer lugar, debido a que la historia de la construcción es omitida.

En este orden de ideas, algo análogo habría que afirmar en relación con la enseñanza de la fisicoquímica. De ser así, se encontraría una explicación de la distribución de quienes aspiraban a estudiar profesionalmente química en Bélgica: los investigadores concluyeron que el $35 \%$ de los aspirantes mostraban preferencia por la química orgánica, y el $61 \%$ por la bioquímica. Comprobaron que tenían actitudes negativas hacia la fisicoquímica, esto es, casi ninguno de los estudiantes se deciden por estudiar esta disciplina (Neerinck y Palmer, 1979). ¿La necesidad de una formación matemática en cálculo diferencial?, ¿una formación matemática que sólo en estos días cambió con la creación del campo de investigación reconocido como educación matemática? o los profesores de la asignatura de fisicoquímica no han accedido al convencimiento de que antes de presentar el desarrollo matemático que la sustenta, habría indispensablemente que coadyuvarlos para la construcción de un modelo o la necesaria representación mental, en la que las ecuaciones adquieren sentido.

\section{Bibliografía}

Bachelard, G. (1976). El materialismo racional. Buenos Aires: Paidós.

Bensaude-Vincent, B. (1991). Lavoisier: una revolución científica. En: Historia de las ciencias, Serres, M. (ed.), pp. 410-435. Madrid: Cátedra.

Bensaude-Vincent, B. y Stengers, I. (1997). Historia de la química. Madrid: AddisonWesley.

Bowler, P. J. y Morus, I. (2007). Panorama general de la ciencia moderna. Barcelona: Crítica.

Brock, W. H. (1998). Historia de la química. Madrid: Alianza.

Fara, P. (2009). Breve historia de la ciencia. Madrid: Ariel.

Gallego Badillo, R., Pérez Miranda, R. y Figueroa Molina, R. (2011). Matematización de los procesos químicos. Primera Parte. Tecné, Episteme y Didaxis, No. 27, 94-107.

Gallego Badillo, R., Pérez Miranda, R. y Gallego Torres A. P. (2009). Una aproximación histórico-epistemológica a las leyes fundamentales de la química. Revista Electrónica de Enseñanza de la Ciencia, Vol. 8, No. 1, Art. 19, 359-375.

Gallego Badillo, R., Pérez Miranda, R., Gallego Torres, A. P. y Torres de Gallego, L. N. (2006). El objeto de saber de los químicos. Formulación, modificación y abandono del modelo icónico inicial. Investigações em Ensino de Ciências, Vol. 11, No. 3, 365-381. 
Matematización de los procesos químicos. Segunda parte Rómulo Gallego Badillo | Royman Pérez Miranda | Adriana Patricia Gallego Torres

Ihde, A. J. (1984). The development of modern chemistry. New York: Dover.

Izquierdo, M. (1988). La contribución de la teoría del flogisto a la estructuración actual de la ciencia química. Implicaciones didácticas. Enseñanza de las Ciencias, 6(1), 67-74.

Jensen, W. B. (1998a). Logic, history and the chemistry textbooks I. Does chemistry have a logical structure? Journal of Chemical Education, Vol. 75, No. 6, 679-687.

Jensen, W. B.(1998b). Logic, history and the chemistry textbooks II. Can we unmuddle the chemistry textbook? Journal of Chemical Education, Vol. 75, No. 7, 917-828.

Jensen, W. B. (1998c). Logic, history and the chemistry textbooks III. One chemical revolution or tree? Journal of Chemical Education, Vol. 75, No. 8, 961-969.

Laidler K. J. (1995). The world of physical chemistry. Oxford: Oxford University Press.

Laín, P. y López, J. M. (1963). Panorama histórico de la ciencia moderna. Madrid: Guadarrama.

Lakatos, I. (1983). La metodología de los programas de investigación científica. Madrid: Alianza.

Lévi-Strauss, C. (1964). El pensamiento salvaje. México: Fondo de Cultura Económica.
Lockemann, G. (1960). Historia de la química. México: UTEHA.

Mayr, E. (2006). Por qué es única la biología. Consideraciones sobre la autonomía de una disciplina científica. Buenos Aires: Katz.

Neerinck, D. y Palmer, C. R. (1979). Aspirations and attitude of students in chemistry. Higher Education, 8, 69-87.

Nelson, G. (2002). Teaching chemistry progressively: From substances, to atoms and molecules, to electrons and nuclei. Chemistry Education: Research and Practice in Europe, Vol. 3, No. 2, 215-228.

Scerri, E. R. (1997). Has the periodic table successfully axiomatizad. Erkenntnis, 47, 229-343.

Scerri, E. R (2001). The new philosophy of chemistry and its relevance to chemical education. Chemistry Education: Research and Practice in Europe, Vol. 2, No. 2, 165170.

Scerri, E. R (2003). Philosophical confusion in chemical education research. Journal of Chemical Education, Vol. 8, No. 5, 468-474.

Schneer, C. J. (1975). Mente y materia. Barcelona: Bruguera. 